\title{
Changes in Femoral Posterior Condylar Offset, Tibial Posterior Slope Angle, and Joint Line Height after Cruciate-Retaining Total Knee Arthroplasty
}

\author{
Sang Jun Song, MD, Dae Kyung Bae, MD, Kang Il Kim, MD, and Ho Yeon Jeong, MD \\ Department of Orthopaedic Surgery, Kyung Hee University School of Medicine, Seoul, Korea
}

\begin{abstract}
Purpose: Changes in the femoral posterior condylar offset (PCO), tibial posterior slope angle (PSA), and joint line height (JLH) after cruciateretaining total knee arthroplasty (CR-TKA) were evaluated to determine their influence on the flexion angle.

Materials and Methods: A total of 125 CR-TKAs performed on 110 patients were retrospectively reviewed. Pre- and postoperative PCO, PSA, and JLH were compared using correlation analysis. Independent factors affecting the postoperative flexion angle of the knee were analyzed.

Results: The PCO was $28.2 \pm 2.0 \mathrm{~mm}$ (range, 24.5 to $33.1 \mathrm{~mm}$ ) preoperatively and $26.7 \pm 1.8 \mathrm{~mm}$ (range, 22.2 to $31.2 \mathrm{~mm}$ ) postoperatively (r=0.807, $\mathrm{p}<0.001$ ). The PSA was $10.4^{\circ} \pm 4.9^{\circ}$ (range, $1.6^{\circ}$ to $21.2^{\circ}$ ) preoperatively and decreased to $4.9^{\circ} \pm 2.0^{\circ}\left(2.2^{\circ}\right.$ to $\left.10.7^{\circ}\right)$ postoperatively $(\mathrm{r}=-0.023, \mathrm{p}=0.800$ ). The JLH was $16.2 \pm 3.0 \mathrm{~mm}$ (range, 10.2 to $27.5 \mathrm{~mm}$ ) preoperatively and $16.1 \pm 2.6 \mathrm{~mm}$ (range, 11.1 to $24.8 \mathrm{~mm}$ ) postoperatively $(\mathrm{r}=0.505$, $\mathrm{p}<0.001$ ). None of the independent factors affected the flexion angle $(\mathrm{p}>0.291)$.

Conclusions: Although the PCO and JLH did not change significantly after CR-TKA, the PSA decreased by $5.5^{\circ}$ with a small range of variation. Restoration of the PCO and JLH could promote optimization of knee flexion in spite of the decreased PSA after CR-TKA.
\end{abstract}

Keywords: Knee, Arthroplasty, Femoral posterior condylar offset, Tibial posterior slope angle, Joint line height

\section{Introduction}

One of the important goals of total knee arthroplasty (TKA) is to increase the functional flexion angle without creating instability. The range of flexion after TKA is probably influenced by many factors, including patient factors, prosthesis design, surgical technique, and rehabilitation. In terms of patient factors, the preoperative flexion angle is known to greatly influence the postoperative flexion angle ${ }^{1,2}$. In terms of surgical factors, changes in the femoral posterior condylar offset (PCO), tibial posterior slope

Received July 27, 2015; Revised (1st) September 1, 2015;

(2nd) October 25, 2015; Accepted January 18, 2016

Correspondence to: Dae Kyung Bae, MD

Department of Orthopaedic Surgery, Kyung Hee University School of Medicine, 26 Kyungheedae-ro, Dongdaemun-gu, Seoul 02447, Korea Tel: +82-2-958-8366, Fax: +82-2-964-3865

E-mail: tesstore@empas.com

This is an Open Access article distributed under the terms of the Creative Commons Attribution Non-Commercial License (http://creativecommons.org/licenses/by-nc/4.0/) which permits unrestricted non-commercial use, distribution, and reproduction in any medium, provided the original work is properly cited. angle (PSA), joint line height (JLH), femoral roll back, posterior cruciate ligament (PCL) tension, gap balancing, and patellar tracking have been analyzed ${ }^{3-8)}$. However, the effects of the PCO, PSA, and JLH on the postoperative flexion angle after TKA are still debated ${ }^{4-6,9-11)}$, and the identification of independent factors that affect the postoperative flexion angle is mostly difficult because many variables are involved ${ }^{5}$.

Restoration of the PCO and PSA has been shown to delay tibiofemoral impingement and increase the flexion angle. Bellemans et al. ${ }^{6)}$ reported that the magnitude of PCO was found to correlate with the flexion angle after cruciate-retaining TKA (CR-TKA). Other previous studies ${ }^{4,12)}$ argued that it did not correlate with the flexion angle because there were lots of variables affecting the flexion angle and a systematic analysis might be difficult to detect the complex effects of confounding variables with conventional radiographic measurements. It is difficult to perform restoration of the PCO and PSA in every knee because of mismatching between the component and bone geometry, flexion gap tightness, and wide variation in the preoperative $\mathrm{PSA}^{1,13}$. We thought that it would be necessary to evaluate the change of PSA, PCO, and JLH 
and to analyze their influences on the postoperative flexion angle concomitantly.

The purposes of this study were to evaluate the changes in the PCO, PSA, and JLH after CR-TKA and to analyze the influence of independent factors on the postoperative flexion angle of the knee. We hypothesized that the PCO, PSA, and JLH would not change significantly after CR-TKA and that several independent factors would affect the postoperative flexion angle ${ }^{6}$.

\section{Materials and Methods}

\section{Subjects}

All patients who underwent CR-TKA with a single type of prosthesis between August 2007 and July 2009 were retrospectively reviewed. During this period, 125 CR-TKAs were performed on 110 patients. Three patients were lost to follow-up before postoperative 2 years, leaving 107 patients (122 TKAs) available for review. Informed consent was obtained from all patients before the review, and no patient refused to participate in this study. The inclusion criterion was primary TKA using the PFC Sigma CR prosthesis (Johnson \& Johnson, Raynham, MA, USA) because of degenerative osteoarthritis. The exclusion criteria were TKA using other prostheses, PCL-substituting TKA, or TKA due to secondary osteoarthritis, hemophilic arthropathy, or partial ankylosis with a range of motion (ROM) of $<90^{\circ}$. The average age of the patients (90 females and 17 males) was 67.2 years (range, 50 to 81 years). The average body mass index was $25.4 \mathrm{~kg} / \mathrm{m}^{2}$ (range, 18.7 to $36.0 \mathrm{~kg} / \mathrm{m}^{2}$ ). Fifty-seven right knees and 65 left knees were treated. The follow-up period averaged 5.8 years (range, 2.0 to 7.9 years).

\section{Methods}

Clinical and radiographic results obtained from these patients were thoroughly evaluated. Patients were contacted on a regular basis and postoperative examinations were performed at 3 months, 6 months, and 1 year after surgery, and annually thereafter. Data were collected from the chart review and radiographs.

The Knee Society knee score and function score were used to evaluate pain and function, including ROM, in the preoperative and latest follow-up sessions. The flexion angle was measured using a long-armed goniometer. Changes in the Knee Society knee and function scores and the flexion angle at the latest follow-up versus baseline were evaluated with paired $t$-tests.

Preoperative and postoperative mechanical axes (MAs) were measured on full-length hip-knee-ankle, weight-bearing anteroposterior (AP) radiographs of the leg (orthoroentgenogram).
The MA for coronal plane alignment was defined as the angle between the femoral and tibial MAs. Serial preoperative and postoperative AP and lateral views of the knee were obtained and reviewed to assess limb alignment, component positioning, PCO, PSA, and $\mathrm{JLH}^{1,6}$. A detailed analysis of the radiographs was performed to determine the femoral angle $(\alpha)$, tibial angle $(\beta)$, femoral flexion angle $(\gamma)$, and tibial PSA $(\delta)$ using the Knee Society roentgenographic evaluation method ${ }^{14)}$. Pre- and postoperative true lateral views of the knee, in which the distal and posterior femoral condyles overlapped exactly, were obtained. Radiographic measurements of the PCO and PSA were taken on these lateral radiographs using a picture archiving and communication system. The PCO was defined as the thickness of the posterior condyle, projected posteriorly to the tangent of the posterior cortex of the femoral shaft (Fig. 1). Pre- and postoperative PCOs were compared after correction for magnification using a reference measurement of the diameter of the femoral shaft $10 \mathrm{~cm}$ proximal to the femoral articular surface ${ }^{6}$. The reference line of the PSA was defined as the line connecting the center of the medullary canal 10 and $20 \mathrm{~cm}$ distal to the tibial plateau (Fig. $2)^{1)}$. The preoperative PSA was defined as the angle between the perpendicular line of reference and a line connecting the anterior and posterior borders of the medial tibial plateau. The postoperative PSA was defined as the angle between the perpendicular line of reference and the undersurface of the tibial component. The JLH was defined as the shortest distance between the fibular

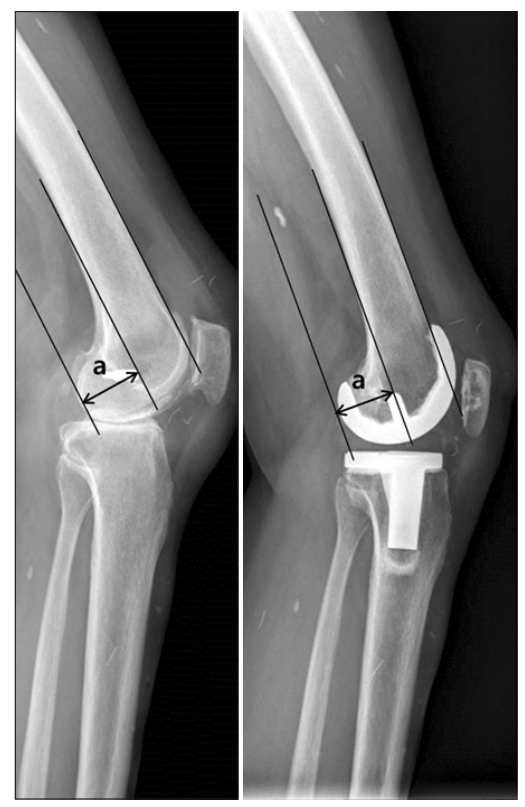

Fig. 1. The measuring method of the femoral posterior condylar offset (a). 


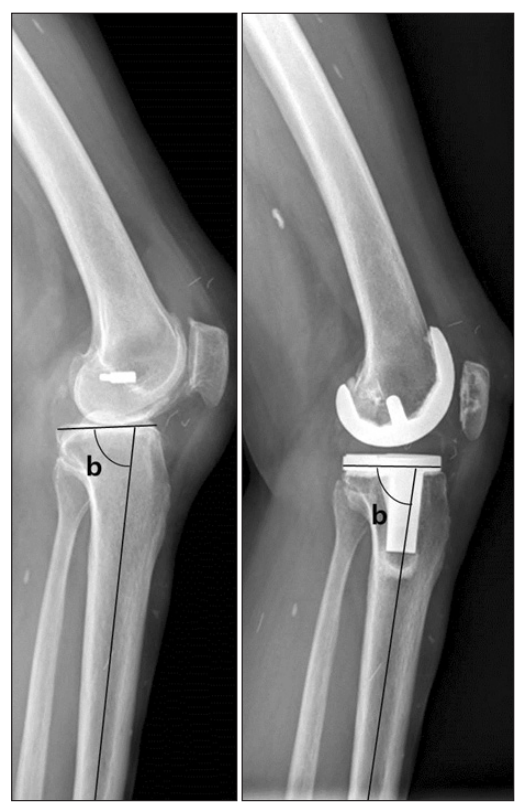

Fig. 2. The measuring method of the tibial posterior slope angle by proximal tibial medullary canal-reference. It was calculated as $90^{\circ}$ (b).

head and lateral femoral condyle. It was measured as the distance between a line perpendicular to the mechanical axis of the tibia at the apex of the fibular head and a parallel line to the first line at the level of the distal aspect of the lateral femoral condyle (Fig. 3).

In addition, the postoperative flexion angle and the amount of increase therein with regard to changes in the PCO, PSA, and JLH were analyzed (Pearson's correlation analysis) ${ }^{2}$. Knees showing postoperative $\mathrm{PCO}$ reduction within $3 \mathrm{~mm}$ of the preoperative value were assigned to the inlier group (PCO-inlier, $\mathrm{n}=111)$. The other 11 knees were assigned to the outlier group (PCO-outlier). There was no knee with an increase in PCO postoperatively. The cut-off value of $3 \mathrm{~mm}$ was selected with the consideration about standard deviation of PCO change in previous studies and the radiographic measurement accuracy ${ }^{4,12}$. Changes in the flexion angle were compared between the PCO-inlier and PCO-outlier groups. Knees within $3^{\circ}$ of the average postoperative PSA of $4.9^{\circ}$ were assigned to the inlier group ${ }^{13,15-17)}$ (PSA-inlier, $\mathrm{n}=108$ ), in which the postoperative PSA ranged from $1.9^{\circ}$ to $7.9^{\circ}$. Fourteen knees were assigned to the outlier group, in which postoperative PSA ranged from $0.2^{\circ}$ to $1.9^{\circ}$.

To reduce observational bias, two independent investigators repetitively performed all radiographic measurements. The intra- and interobserver reliabilities of all measurements were assessed using the intraclass correlation coefficient (ICC). These reliabilities were confirmed by ICC values exceeding 0.85 for all measurements (Pearson's correlation analysis).

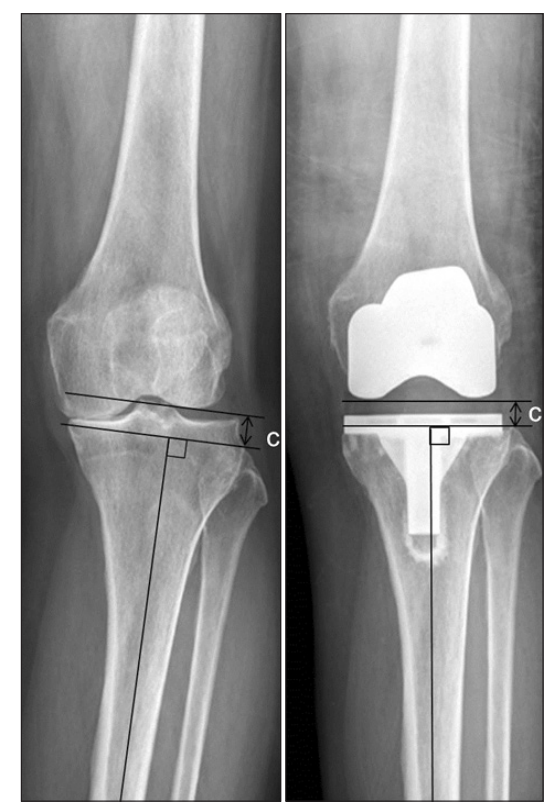

Fig. 3. The measuring method of the joint line height. The distance (c) is measured between a line perpendicular to the mechanical axis of the tibia at the apex of the fibular head and a parallel line to the first line at the level of the distal aspect of the lateral femoral condyle.

\section{Surgical Technique}

All TKAs were performed by the single surgeon (Bae) using a midline skin incision and medial parapatellar arthrotomy. A tourniquet was used in all knees. Bone cuts were made using a modified measured resection technique. The size of the femoral component was selected using the anterior-referencing method, considering the frequent flexion tightness during CR-TKA ${ }^{18)}$ and relatively high $\mathrm{AP} / \mathrm{ML}$ ratio of the used femoral component (PFC Sigma). For cases in which the AP dimension of the femoral condyles fell between size grades, the smaller femoral component was used to avoid the tendency of flexion gap tightness in CR$\mathrm{TKA}^{18)}$. However, efforts were made to reduce the alteration of PCO by shifting the AP cutting guide $2 \mathrm{~mm}$ backward using Sigma instrument. In cases of mediolateral overhang, the size of the femoral component was reduced by one grade. Thirty two knees were downsized due to between size grades of the femoral component, and three knees were downsized due to mediolateral overhang. The extramedullary tibial cutting guide was used. The tibial slope was usually set to $3^{\circ}$ of the posterior slope in the sagittal plane. All osteophytes were removed. Any contracted medial or lateral soft tissue was evaluated carefully with palpation and then released selectively. The flexion and extension gaps were also assessed with a spacer block or trial component. In cases of flexion tightness or lift-off and/or disturbance of rollback during 
flexion, the PCL was released or the posterior slope of the tibia was increased as necessary. The tibial cut surface was trimmed and adjusted using a sharp electronic saw with confirmation of sagittal alignment. All patellae were resurfaced. All components were cemented onto cleansed and dried surfaces. There was no knee in which it was necessary to manipulate under anesthesia because of postoperative ROM limitation.

\section{Statistical Analysis}

Statistical analyses were performed using SPSS ver. 18.0 (SPSS Inc., Chicago, IL, USA). The pre- and postoperative PCO, PSA, and JLH were compared using correlation analysis ${ }^{19)}$. The changes in the flexion angle were compared between the PCOinlier and PCO-outlier groups using Student's $t$-test. They were also compared between the PSA-inlier and PSA-outlier groups (Student's $t$-test). The correlation between the preoperative flexion angle and the preoperative PCO or PSA was analyzed. The correlation between the preoperative PCO and PSA was also analyzed. A p-value of $<0.05$ was considered to indicate statistical significance.

\section{Results}

\section{Clinical Results}

The average Knee Society knee score was $55.1 \pm 10.9$ before surgery and $93.7 \pm 3.4$ at the final follow-up examination $(\mathrm{p}<0.001)$. The average function score was $55.4 \pm 9.3$ before surgery and $92.0 \pm 4.8$ at the final follow-up examination $(\mathrm{p}<0.001)$. The average flexion contracture was $5.1^{\circ} \pm 6.3^{\circ}$ before surgery and $0.6^{\circ} \pm 2.1^{\circ}$ at the final follow-up examination ( $\mathrm{p}<0.001)$. The average further flexion was $133.2^{\circ} \pm 8.6^{\circ}$ before surgery and $132.9^{\circ} \pm 6.9^{\circ}$ at the final follow-up examination $(\mathrm{p}=0.200)$.

\section{Radiographic Results}

The average MA was $10.0^{\circ} \pm 4.9^{\circ}$ varus preoperatively and $0.3^{\circ} \pm 1.8^{\circ}$ varus postoperatively. According to the roentgeno-

Table 1. Correlations between Preoperative and Postoperative PCO, PSA, and JLH after Cruciate-Retaining Total Knee Arthroplasty

\begin{tabular}{lrrcr}
\hline Variable & Preoperative & Postoperative & $\begin{array}{c}\text { Correlation } \\
\text { coefficient }\end{array}$ & p-value \\
\hline PCO $(\mathrm{mm})$ & $28.2 \pm 2.0$ & $26.7 \pm 1.8$ & 0.807 & $<0.001$ \\
$\mathrm{PSA}\left({ }^{\circ}\right)$ & $10.4 \pm 4.9$ & $4.9 \pm 2.0$ & -0.023 & 0.800 \\
$\mathrm{JLH}(\mathrm{mm})$ & $16.1 \pm 3.0$ & $16.0 \pm 2.6$ & 0.505 & $<0.001$ \\
\hline
\end{tabular}

PCO: posterior condylar offset, PSA: posterior slope angle, JLH: joint line height. graphic evaluation method of the American Knee Society, the mean $\alpha, \beta, \gamma$, and $\delta$ angles were $95.8^{\circ} \pm 0.9^{\circ}, 90.2^{\circ} \pm 0.9^{\circ}, 2.1^{\circ} \pm 1.1^{\circ}$, and $85.2^{\circ} \pm 2.2^{\circ}$, respectively. The average PCO was $28.2 \pm 2.0$ $\mathrm{mm}$ preoperatively and $26.7 \pm 1.8 \mathrm{~mm}$ postoperatively $(\mathrm{r}=0.807$, $\mathrm{p}<0.001)$. The average PSA was $10.4^{\circ} \pm 4.9^{\circ}$ preoperatively and $4.9^{\circ} \pm 2.0^{\circ}$ postoperatively $(\mathrm{r}=-0.023, \mathrm{p}=0.800)$. The average $\mathrm{JLH}$ was $16.2 \pm 3.0 \mathrm{~mm}$ preoperatively and $16.1 \pm 2.6 \mathrm{~mm}$ postoperatively $(\mathrm{r}=0.505, \mathrm{p}<0.001)$ (Table 1$)$.

\section{Correlation between Changes in PCO, PSA, and JLH and the Change in Flexion Angle}

Changes in the PCO, PSA, and JLH after TKA did not influence the postoperative flexion angle $(\mathrm{p}=0.878,0.715$, and 0.486 , respectively) or the change therein ( $\mathrm{p}=0.486,0.146$, and 0.540 , respectively). The average postoperative flexion angle and change therein did not differ significantly between the PCO-inlier and PCO-outlier groups ( $p=0.323$ and 0.571 , respectively). The average postoperative flexion angle did not differ significantly between the PSA-inlier and PSA-outlier groups ( $\mathrm{p}=0.986$ ). The average flexion angle increased by $0.9^{\circ} \pm 11.9^{\circ}$ in the PSA-inlier group, but decreased by $0.6^{\circ} \pm 6.8^{\circ}$ in the PSA-outlier group; however, this difference was not significant $(\mathrm{p}=0.718)$. The only independent factor that affected the postoperative flexion angle was the preoperative flexion angle $(\mathrm{r}=0.541, \mathrm{p}=0.039)$.

The preoperative $\mathrm{PCO}$ was significantly correlated with the preoperative flexion angle $(r=0.226, p=0.012)$, but the preoperative PSA was not $(\mathrm{p}=0.067)$. The preoperative PCO was significantly correlated with the preoperative PSA $(r=-0.362, \mathrm{p}<0.001)$.

\section{Discussion}

The most important finding of the present study was that the PCO and JLH did not change significantly after CR-TKA. However, the postoperative PSA decreased by as much as $5.5^{\circ}$ on average, with a small range of variation. The average amount of decrease in the PCO after TKA was $1.5 \mathrm{~mm}$, despite the use of an anterior reference guide system in the present study. We used the PFC Sigma instrument, in which PCO alteration can be reduced by adjusting the drill guide position and size of cutting block. The sizes of the femoral components used were 1.5, $2,2.5$, and 3 , which are separated by $2-3-\mathrm{mm}$ increments in the AP dimension. Although femoral components cannot exactly match femoral size in every knee, the authors' efforts to reduce PCO alteration by shifting the AP cutting guide slightly backward reduced the decrease in the PCO. The restoration of normal JLH is important to regain normal knee kinematics and increase 
mid-flexion stability $\mathrm{y}^{20,21)}$. The change in the JLH was $0.1 \mathrm{~mm}$ in the present study, which employed careful determination of the femoral and tibial bone resection levels and confirmation with caliper-obtained measurements of resected condylar thickness ${ }^{22)}$. The change in the PSA was larger in the present study than in other studies ${ }^{5,13)}$. Our preoperative PCO and PSA data were similar to those of an anatomical study of normal knees in which the PSA exhibited greater variability than the PCO, and the PSA and PCO were correlated ${ }^{23}$. Patients with high preoperative PCOs tended to have small PSAs. We could imagine the concomitant effect of PCO and PSA on the individual flexion angle.

Although the preoperative PCO was significantly correlated with preoperative flexion angle in the present study, there are lots of factors affecting the flexion angle. Degenerative changes around the knee joint including osteophytes and loose bodies could also influence the flexion angle as mechanical obstacles. However, they were removed clearly during TKA procedure and those qualified variables could not be evaluated in the present study.

A possible reason for the decrease in the PSA after TKA might be the wide variation in preoperative PSA values. While the variation in preoperative PCO and JLH values was small (Table 1), the standard deviation of the preoperative PSA was $4.9^{\circ}$ and the values ranged from $1.6^{\circ}$ to $21.2^{\circ}$. Several authors ${ }^{1,23)}$ have also reported variable ranges of anatomical PSA in normal knees. The restoration of the PSA after TKA has been shown to delay tibiofemoral impingement, decrease the PCL load, enhance femoral roll back, and improve the flexion angle of the knee ${ }^{24,25}$. However, an excessive PSA in TKA has potentially deleterious effects ${ }^{15,26)}$. It may induce AP instability, cause anterior subluxation of the tibial component, move the joint contact force location to eccentric positions, increase the wear on the posterior portion of the polyethylene surface, and cause aseptic loosening ${ }^{27-29)}$. Because the posterior horn of the meniscus is thicker than the anterior horn, the biomechanical PSA determined with consideration of this structural feature differed from bony PSA measured on radiographs $^{30)}$. A previous study ${ }^{16)}$ demonstrated the importance of the lateral PSA as a guide in order not to cut the tibia at more than $8^{\circ}$. We thought that the restoration of the original PSA in every case is impossible because of the high degree and wide range of variation in the preoperative PSA, and the excessively steep slope doesn't have to be restored with the consideration of the possible various complications ${ }^{27-29)}$. Postoperative PSAs are recommended variously according to prosthesis type due to diversity in the posterior offset of the femoral component and the slope of the polyethylene insert ${ }^{1)}$. The proper cutting angle for the PSA is still controversial, and a wide range of optimal angles has been proposed $^{1)}$. Generally, the PSA can be more reasonably set to $3^{\circ}$ to $5^{\circ}$ of the posterior slope in the sagittal plane during the resection of the proximal tibia, and the tibial cut surface should be adjusted using a sharp electronic saw after confirmation of flexion and extension gap balancing, PCL tension, and femoral roll back ${ }^{11}$.

Another important finding of the present study is that changes in the PCO, PSA, and JLH did not affect the postoperative flexion angle after CR-TKA. The only independent factor that affected the postoperative flexion angle was the preoperative flexion angle. There have been lots of previous studies about the impact of PCO and PSA on the postoperative flexion angle ${ }^{1,2,4,6)}$. It was reported that the postoperative PCO and PSA influenced the postoperative flexion angle in some studies ${ }^{2,6)}$ or they did not in other studies ${ }^{1,4,17}$. The restoration of the PCO delays tibiofemoral impingement and increases knee flexion, especially in cases with paradoxical femoral roll-forward ${ }^{6,25)}$. However, the postoperative flexion angle is influenced by various factors, such as the severity of preoperative deformity, flexion angle of the femoral component, alteration of the PSA and flexion gap, PCL tension, softtissue balance, type of prosthesis, and rehabilitation ${ }^{2,3}$. Thus, the comparative analysis of clinical outcomes according to postoperative PCO is complicated by inherent statistical bias. Furthermore, the alteration in the postoperative PCO was small (average, -1.5 $\mathrm{mm}$ ) in the present study, and only 11 outliers showed a decrease of more than $3 \mathrm{~mm}$. Thus, the comparison of the flexion angle in relation to the postoperative PCO should be carefully interpreted. The comparison of the flexion angle in relation to the postoperative JLH and PSA should also be carefully interpreted for similar reasons.

This study was also limited by its retrospective design and lack of a control group. Most patients were women, and all had osteoarthritic knees with varus deformities. This female predominance in the distributions of osteoarthritis and varus deformity is common in our populations because of the differences in disease demographics among ethnic groups and gender differences in the incidence of bowed legs. Another limitation was that the femoral component size and final PSA were determined by the prediction and experience of the corresponding author. However, the surgeon already had the sufficient experience of TKA (more than 200 TKAs annually), and his decision for sizing and balancing could improve the quality of our data. The strengths of the present study include the prospective collection of a single surgeon's data and minimal loss to follow-up despite the retrospective nature of the study. 


\section{Conclusions}

The PCO and JLH did not change significantly after CR-TKA in the present study. The PSA decreased by $5.5^{\circ}$ with a small range of variation. Those amounts of their changes did not affect the postoperative flexion angle after CR-TKA in the present study.

The Knee Society knee and function scores were satisfactory and the mean further flexion exceeded $130^{\circ}$, with admitted PSA alteration after CR-TKA. Restoration of the PCO and JLH could make the optimization of knee flexion in spite of the decreased PSA after CR-TKA. It is questionable whether surgeons should always restore the original PSA despite the wide range in this angle.

\section{Conflict of Interest}

No potential conflict of interest relevant to this article was reported.

\section{References}

1. Bae DK, Song SJ, Yoon KH, Noh JH, Moon SC. Comparative study of tibial posterior slope angle following cruciateretaining total knee arthroplasty using one of three implants. Int Orthop. 2012;36:755-60.

2. Fujimoto E, Sasashige Y, Masuda Y, Hisatome T, Eguchi A, Masuda T, Sawa M, Nagata Y. Significant effect of the posterior tibial slope and medial/lateral ligament balance on knee flexion in total knee arthroplasty. Knee Surg Sports Traumatol Arthrosc. 2013;21:2704-12.

3. Jia YT, Wang L, Zhang Y, Zhao C, Sun ZH, Liu J. Does mismatch of the femoral component aspect ratio influence the range of knee flexion after posterior-stabilized total knee arthroplasty? Chin J Traumatol. 2012;15:152-7.

4. Seo SS, Ha DJ, Kim CW, Choi JS. Effect of posterior condylar offset on cruciate-retaining mobile TKA. Orthopedics. 2009;32(10 Suppl):44-8.

5. Bellemans J, Robijns F, Duerinckx J, Banks S, Vandenneucker $\mathrm{H}$. The influence of tibial slope on maximal flexion after total knee arthroplasty. Knee Surg Sports Traumatol Arthrosc. 2005;13:193-6.

6. Bellemans J, Banks S, Victor J, Vandenneucker H, Moemans A. Fluoroscopic analysis of the kinematics of deep flexion in total knee arthroplasty: influence of posterior condylar offset. J Bone Joint Surg Br. 2002;84:50-3.

7. Dennis DA, Kim RH, Johnson DR, Springer BD, Fehring
TK, Sharma A. The John Insall Award: control-matched evaluation of painful patellar Crepitus after total knee arthroplasty. Clin Orthop Relat Res. 2011;469:10-7.

8. Del Gaizo DJ, Della Valle CJ. Instability in primary total knee arthroplasty. Orthopedics. 2011;34:e519-21.

9. Kansara D, Markel DC. The effect of posterior tibial slope on range of motion after total knee arthroplasty. J Arthroplasty. 2006;21:809-13.

10. Kim JH. Effect of posterior femoral condylar offset and posterior tibial slope on maximal flexion angle of the knee in posterior cruciate ligament sacrificing total knee arthroplasty. Knee Surg Relat Res. 2013;25:54-9.

11. Youm YS, Cho SD, Eo J, Park KB, Lee SH. Can we use the posterior condylar off set as a predictive factor for overhang of the implant in total knee arthroplasty? Knee Surg Relat Res. 2011;23:149-52.

12. Ishii Y, Noguchi H, Takeda M, Sato J, Toyabe S. Posterior condylar offset does not correlate with knee flexion after TKA. Clin Orthop Relat Res. 2013;471:2995-3001.

13. de Boer JJ, Blankevoort L, Kingma I, Vorster W. In vitro study of inter-individual variation in posterior slope in the knee joint. Clin Biomech (Bristol, Avon). 2009;24:488-92.

14. Ewald FC. The Knee Society total knee arthroplasty roentgenographic evaluation and scoring system. Clin Orthop Relat Res. 1989;(248):9-12.

15. Wasielewski RC, Galante JO, Leighty RM, Natarajan RN, Rosenberg AG. Wear patterns on retrieved polyethylene tibial inserts and their relationship to technical considerations during total knee arthroplasty. Clin Orthop Relat Res. 1994;(299):31-43.

16. Kuwano T, Urabe K, Miura H, Nagamine R, Matsuda S, Satomura M, Sasaki T, Sakai S, Honda H, Iwamoto Y. Importance of the lateral anatomic tibial slope as a guide to the tibial cut in total knee arthroplasty in Japanese patients. J Orthop Sci. 2005;10:42-7.

17. Shi X, Shen B, Kang P, Yang J, Zhou Z, Pei F. The effect of posterior tibial slope on knee flexion in posterior-stabilized total knee arthroplasty. Knee Surg Sports Traumatol Arthrosc. 2013;21:2696-703.

18. In Y, Kim JM, Woo YK, Choi NY, Sohn JM, Koh HS. Factors affecting flexion gap tightness in cruciate-retaining total knee arthroplasty. J Arthroplasty. 2009;24:317-21.

19. Hsu WH, Hsu RW, Weng YJ. Effect of preoperative deformity on postoperative leg axis in total knee arthroplasty: a prospective randomized study. Knee Surg Sports Traumatol Arthrosc. 2010;18:1323-7. 
20. Porteous AJ, Hassaballa MA, Newman JH. Does the joint line matter in revision total knee replacement? J Bone Joint Surg Br. 2008;90:879-84.

21. Cope MR, O'Brien BS, Nanu AM. The influence of the posterior cruciate ligament in the maintenance of joint line in primary total knee arthroplasty: a radiologic study. J Arthroplasty. 2002;17:206-8.

22. Bae DK, Song SJ, Yoon KH, Noh JH, Lee CH. Intraoperative assessment of resected condyle thickness in total knee arthroplasty. Knee Surg Sports Traumatol Arthrosc. 2012;20:2039-46.

23. Cinotti G, Sessa P, Ripani FR, Postacchini R, Masciangelo R, Giannicola G. Correlation between posterior offset of femoral condyles and sagittal slope of the tibial plateau. J Anat. 2012;221:452-8.

24. Banks S, Bellemans J, Nozaki H, Whiteside LA, Harman M, Hodge WA. Knee motions during maximum flexion in fixed and mobile-bearing arthroplasties. Clin Orthop Relat Res. 2003;(410):131-8.

25. Massin P, Gournay A. Optimization of the posterior condylar offset, tibial slope, and condylar roll-back in total knee arthroplasty. J Arthroplasty. 2006;21:889-96.

26. Migaud H, De Ladoucette A, Dohin B, Cloutier JM, Gougeon F, Duquennoy A. Influence of the tibial slope on tibial translation and mobility of non-constrained total knee prosthesis. Rev Chir Orthop Reparatrice Appar Mot. 1996;82:713.

27. Jojima H, Whiteside LA, Ogata K. Effect of tibial slope or posterior cruciate ligament release on knee kinematics. Clin Orthop Relat Res. 2004;(426):194-8.

28. Catani F, Leardini A, Ensini A, Cucca G, Bragonzoni L, Toksvig-Larsen S, Giannini S. The stability of the cemented tibial component of total knee arthroplasty: posterior cruciate-retaining versus posterior-stabilized design. J Arthroplasty. 2004;19:775-82.

29. Bai B, Baez J, Testa N, Kummer FJ. Effect of posterior cut angle on tibial component loading. J Arthroplasty. 2000;15:91620.

30. Jenny JY, Rapp E, Kehr P. Proximal tibial meniscal slope: a comparison with the bone slope. Rev Chir Orthop Reparatrice Appar Mot. 1997;83:435-8. 